\title{
ON THE POWER SERIES FOR ELLIPTIC FUNCTIONS*
}

BY

E. T. BELL

1. Introduction. A more direct and more practicable method than those hitherto used for obtaining the coefficients in the power series expansions of doubly periodic and other elliptic theta quotients appears incidentally in some work relating to representations of rational integers as sums of integer squares. References to the literature will be found in the paper of Grudert, and in the treatises of Enneper $\ddagger$ and Krause. $\S$ Most of the complications in some other methods, for example that of $\mathrm{D}$. Andréll, enter with the use of the differential equation (or the equivalent difference equation, obtained by equating coefficients) satisfied by the function to be expanded. By avoiding the use of the differential equation entirely, the arithmetical nature of the coefficients in the power series becomes evident, and much tedious algebra is obviated. In the method used here the difference equations for the coeffcients are linear; other methods introduce non-linear equations.

Hermite $\int$ proposed an extremely ingenious and elegant method, based on the transformation of the second order, for obtaining the coefficients when the functions are doubly periodic. Later** he remarked that this method is incapable, apparently, of leading to the desired end. However, Gruder (loc. cit., pp. 158-166) succeeded in obtaining explicit formulas for certain coefficients by this method, although the arithmetical character of the coefficients is perhaps not as evident as it might be.

Hermite** published only specimens of the results furnished by another method, without indicating what this method was. As noted by Picard $\dagger \dagger$, some of Hermite's explicit formulas thus obtained are incorrect (owing to

* Presented to the Society, September 7, 1934; received by the editors April 30, 1934.

$\dagger$ O. Gruder, Wiener Sitzungsberichte, II a, vol. 126 (1917), pp. 125-183.

$\ddagger$ A. Enneper, Elliptische Functionen, 1890, \$47.

$\$$ M. Krause, Theorie der doppeltperiodischen Functionen, 1895, $\$ 43$.

|| D. André, Annales de l'École Normale Supérieure, (2), vol. 6 (1877), pp. 265-328. Ibid., vol. 8 (1879), pp. 151-168; vol. 9 (1880), pp. 107-118.

T Ch. Hermite, Comptes Rendus (Paris), vol. 57 (1863), pp. 613-618; Liouville's Journal, (2), vol. 9 (1864), pp. 289-295.

** Ch. Hermite, Lettre d M. Königsberger, Crelle's Journal, vol. 81 (1876), pp. 220-228. Oeuvres, vol. 3, pp. 236-245. See also Oeuvres, vol. 3, pp. 222-231.

†† Hermite, Oeuvres, vol. 3, p. 237. 
slips in calculation). The incorrect formulas have been reproduced in the treatises by Enneper and Krause cited above; they may be easily corrected by the present method. The expansion of $\mathrm{cn} x$ being one of those containing errors, we shall consider it first in detail as an illustration of the general method, which consists of comparing the MacLaurin and Fourier expansions of the function whose power series expansion is sought. If the origin is a singularity of the function, the singularity is removed by any of the familiar devices used in obtaining the Fourier expansion; the procedure will be clear from the examples in $\$ \S 4,5$. All of the series in the sequel are absolutely convergent for values of the variables different from zero.

2. Expansion of $\mathrm{cn} x$. It is readily seen that the expansion is of the form

$$
\begin{gathered}
\text { cn } x=1+\sum_{c=1}^{\infty}(-1)^{\circ} Q_{s}\left(k^{2}\right) \frac{x^{2}}{(2 s) !}, \\
Q_{\bullet}\left(k^{2}\right) \equiv \sum_{r=0}^{-1} q_{r}(s) k^{2 r},
\end{gathered}
$$

where the $q_{r}(s)$ are integers. The problem of expanding $\mathrm{cn} x$ is thus reduced to that of calculating $q_{r}(s)$ as a function of $r, s$.

Replacing $\mathrm{cn} x$ by its equivalent theta quotient, and expanding the latter in a cosine series, ${ }^{*}$

$$
\vartheta_{2}^{2} \mathrm{cn}\left(x \vartheta_{s}^{2}\right)=\vartheta_{0} \vartheta_{2} \frac{\vartheta_{2}(x)}{\vartheta_{0}(x)}=4 \sum q^{m / 2}\left[\sum(-1 \mid \tau) \cos t x\right],
$$

$m=1,3,5, \cdots ; m=t \tau(t, \tau$ integers $>0),(-1 \mid \tau)=(-1)^{(\tau-1) / 2}$. In the last we now expand the cosines, rearrange the result (as is obviously permissible) as a power series in $x$, apply (1) to the left of (3), and finally equate coefficients of $x^{28}$. Thus

$$
\vartheta_{2}^{2} \vartheta_{3}^{4 *} Q_{\bullet}\left(k^{2}\right)=4 \sum q^{m / 2} \xi_{20}(m),
$$

where $\xi_{2 s}(m)$ denotes the sum of the (2s)th powers of all those (positive) divisors of $m$ whose conjugate divisors are of the form $4 h+1$ minus the like sum in which the conjugates are of the form $4 h+3$. In (4) we apply (2), replace $q$ by $q^{4}$ in the result, and get

$$
\sum_{r=0}^{-1} q_{r}(s) \vartheta_{2}^{4 r+2}\left(q^{4}\right) \vartheta_{3}^{4 r-4 r}\left(q^{4}\right)=4 \sum q^{2 m \xi_{n_{\theta}}(m)}
$$

* This series, with others of a similar kind in later sections, is given with many more in my paper, Messenger of Mathematics, vol. 54 (1924), pp. 116-176. The recurrence (6) occurs incidentally in my paper on sums of squares, Bulletin of the American Mathematical Society, vol. 26 (1919), pp. 19-25. 
Let $N(n, f, g)$ denote the number of those representations of $n$ as a sum of $f$ squares, precisely $g$ of which are odd with roots greater than zero, and occupy the first $g$ places in the representations, and $f-g$ are even with roots greater than, equal to, or less than zero. Then, from (5) and the definition of $N$, we have the following recurrence for the $q_{r}(s)$ :

$$
\sum_{r=0}^{(m-1) / 2} 2^{4 r} N(2 m, 4 s+2,4 r+2) q_{r}(s)=\xi_{2 s}(m) .
$$

To calculate $q_{3}(s)$ we take $m=1,3,5, \cdots, 2 j+1$ in (6) and solve the resulting linear equations for $q_{j}(s)$. An explicit determinant formula for the general coefficient $q_{j}(s)$ is thus obtained, but it is more practical to proceed step by step, evaluating the numbers $N$ as they occur. To illustrate the process, we shall calculate $q_{0}(s), q_{1}(s), q_{2}(s), q_{3}(s)$, the first three of which were given correctly, and the fourth incorrectly, by Hermite.

$m=1$ :

$$
N(2,4 s+2,2) q_{0}(s)=\xi_{2 o}(1) .
$$

Referring to the definition of $N$, we see that $2=1^{2}+1^{2}+4 s \cdot 0^{2}$ is the only representation enumerated by $N(2,4 s+2,2)$. By the definition of $\xi, \xi_{20}(1)=1$. Hence $q_{0}(s)=1$.

$$
\begin{aligned}
& m=3: \quad N(6,4 s+2,2) q_{0}(s)+N(6,4 s+2,6) 2^{4} q_{1}(s)=\xi_{2 s}(3) ; \\
& 6=1^{2}+1^{2}+\left[2^{2}+(4 s-1) 0^{2}\right] \text {, } \\
& N(6,4 s+2,2)=\frac{2(4 s) !}{1 !(4 s-1) !}=8 s ; \quad \xi_{2 s}(3)=3^{2 s}-1 ; \\
& 2^{4} q_{1}(s)=3^{2 s}-8 s-1 \text {. } \\
& m=5 \text { : } \\
& 10=1^{2}+1^{2}+\left[2^{2}+2^{2}+(4 s-2) 0^{2}\right]=1^{2}+3^{2}+\left[4 s\left(0^{2}\right)\right], \\
& N(10,4 s+2,2)=\frac{2^{2}(4 s) !}{2 !(4 s-2) !}+2=2\left(16 s^{2}-4 s+1\right) ; \\
& 10=6 \cdot 1^{2}+\left[2^{2}+(4 s-5) 0^{2}\right] \text {, } \\
& N(10,4 s+2,6)=\frac{2(4 s-4) !}{1 !(4 s-5) !}=8(s-1) ; \\
& N(10,4 s+2,10)=1 ; \xi_{2 s}(5)=5^{20}+1 \text {; } \\
& \left(32 s^{2}-8 s+2\right) q_{0}(s)+8(s-1) 2^{4} q_{1}(s)+2^{8} q_{2}(s)=5^{2 \imath}+1 \text {; } \\
& 2^{8} q_{2}(s)=5^{2 s}-8(s-1) 2^{2 s}+32 s^{2}-48 s-9 .
\end{aligned}
$$


$m=7$ :

$$
\begin{aligned}
& 14=2 \cdot 1^{2}+\left[3 \cdot 2^{2}+(4 s-3) 0^{2}\right]=1^{2}+3^{2}+\left[2^{2}+(4 s-1) 0^{2}\right], \\
& N(14,4 s+2,2)=\frac{2^{3}(4 s) !}{3 !(4 s-3) !}+2 \cdot 2 \cdot \frac{(4 s) !}{1 !(4 s-1) !}, \\
& =\frac{16}{3} s\left(16 s^{2}-12 s+5\right) \text {; } \\
& 14=6 \cdot 1^{2}+\left[2 \cdot 2^{2}+(4 s-6) 0^{2}\right]=3^{2}+5 \cdot 1^{2}, \\
& N(14,4 s+2,6)=\frac{2^{2}(4 s-4) !}{2 !(4 s-6) !}+6=2\left(16 s^{2}-36 s+23\right) \text {; } \\
& 14=10 \cdot 1^{2}+\left[2^{2}+(4 s-9) 0^{2}\right] \\
& N(14,4 s+2,10)=\frac{2(4 s-8) !}{1 !(4 s-9) !}=8(s-2) \text {; } \\
& N(14,4 s+2,14)=1 ; \quad \xi_{2 s}(7)=7^{2 s}-1 ; \\
& 2^{12} q_{3}(s)=7^{2 s}-8(s-2) 5^{2 s}+2\left(16 s^{2}-60 s+41\right) 3^{2 s} \\
& -\frac{1}{3}\left(256 s^{8}-1248 s^{2}+1280 s-297\right) .
\end{aligned}
$$

For $s=1,2,3, \cdots$ these values check with the numerical results given in the treatises. By the transformation of the first order,

$$
\operatorname{sn}(k u, 1 / k)=k \operatorname{sn}(x, k) \text {; }
$$

whence $q_{j}(s)=q_{s-j}(s)$.

From (6) and the definition of $\xi_{2 s}(m)$ it is evident that

(7) ${ }^{24 j} q_{j}(s)=(2 j+1)^{\bullet}+A_{1}(s)(2 j-1)^{\bullet}+A_{2}(s)(2 j-3)^{\bullet}+\cdots+A_{j}(s) 1^{\bullet}$,

where the $A$ 's are polynomials in $s$ with rational coefficients. It will be shown that the degree in $s$ of $A_{r}(s)$ is $r(r=1, \cdots, j)$. The last is an immediate consequence of (6) and the following lemma.

The degree in sof $N(2 m, 4 s+2,2 m-4 h)$ is $h(h=0,1, \cdots,(m-1) / 2)$.

Before proving the lemma we shall examine it for $h=0,1,2,3$. Obviously $N(2 m, 4 s+2,2 m)=1$. For $h=1$ we have, as the only possible decomposition of $2 m$ of the kind enumerated by $N(2 m, 4 s+2,2 m-4)$,

$$
2 m=(2 m-4) 1^{2}+\left[2^{2}+\{(4 s+2)-(2 m-3)\} 0^{2}\right]
$$

hence, enumerating the corresponding representations, we get

$$
N(2 m, 4 s+2,2 m-4)=\frac{2 \cdot(4 s-2 m+6) !}{1 !(4 s-2 m+5) !}=4(2 s-m+3) .
$$


Here a negative result $(m>2 s+3)$ is to be interpreted as zero (no representations), and likewise in all similar cases. When $h=2$, of the $2 m-8$ odd squares in the representations enumerated by $N(2 m, 4 s+2,2 m-8)$ all may be 1 's or precisely one may be $3^{2}$, and there are no other possibilities. Hence the only decompositions to be considered are

$$
2 m=(2 m-8) 1^{2}+\left[2 \cdot 2^{2}+(4 s-2 m+8) 0^{2}\right]=(2 m-9) 1^{2}+3^{2} ;
$$

whence, counting the representations of the kind enumerated by $N$ we have

$$
N(2 m, 4 s+2,2 m-8)=\frac{2^{2} \cdot(4 s-2 m+10) !}{2 !(2 s-2 m+8) !}+\frac{(2 m-8) !}{1 !(2 m-9) !},
$$

the last fraction corresponding to the representations obtained by arranging the $(2 m-9) 1$ 's and the $3^{2}$ in all possible ways. Thus

$$
N(2 m, 4 s+2,2 m-8)=2\left[16 s^{2}-4(3 m-14)+2 m^{2}-18 m+41\right] .
$$

The only decompositions of $2 m$ to be considered when $h=3$ are

$$
\begin{aligned}
2 m & =(2 m-12) 1^{2}+\left[3 \cdot 2^{2}+(4 s-2 m+11) 0^{2}\right], \\
& =(2 m-13) 1^{2}+3^{2}+\left[2^{2}+(4 s-2 m+13) 0^{2}\right] ;
\end{aligned}
$$

whence

$$
\begin{aligned}
N(2 m, 4 s+2,2 m-12)= & \frac{2^{3}(4 s-2 m+14) !}{3 !(4 s-2 m+11) !} \\
& +\frac{(2 m-12) !}{1 !(2 m-13) !} \cdot \frac{2 \cdot(4 s-2 m+14) !}{1 !(4 s-2 m+13) !},
\end{aligned}
$$

which is of degree 3 in $s$.

To prove the lemma, consider the decomposition

$$
2 m=(2 m-4 h) 1^{2}+\left[h \cdot 2^{2}+(4 s+2-2 m+3 h) 0^{2}\right],
$$

which contributes to $N(2 m, 4 s+2,2 m-4 h)$ precisely

$$
A(t) \equiv \frac{2^{h} \cdot(t+4 h) !}{h !(t+3 h) !} \quad(t \equiv 4 s+2-2 m)
$$

representations. If $h \cdot 2^{2}$ has a decomposition into a sum of $t+4 h$ even squares other than that in [ ] above, it is of the form indicated in [ ] in the following decomposition of $2 m$,

$$
2 m=(2 m-4 h) 1^{2}+\left[h_{1} a_{1}^{2}+\cdots+h_{p} a_{p}^{2}+h_{p+1} 0^{2}\right],
$$

where $h_{i}, a_{i}(i=1, \cdots, p)$ are $>0, a_{i} \geqq 2$, the $a_{1}, \cdots, a_{p}$ are distinct, and 
$h_{1}+\cdots+h_{p}+h_{p+1}=t+4 h$. This decomposition contributes to $N(2 m, 4 s+2$, $2 m-4 h)$ precisely

$$
A^{\prime}(t) \equiv \frac{2^{h_{1}+\cdots+h_{p}}(t+4 h) !}{h_{1} ! \cdots h_{p} !\left(t+4 h-h_{1}-\cdots-h_{p}\right) !}
$$

representations. The degree in $t$, and hence also in $s$, of the polynomial $A(t)$ is $h$; that of $A^{\prime}(t)$ is $h_{1}+\cdots+h_{p}$. Hence, when it is shown that $h_{1}+\cdots+h_{p}$ $<h$, the lemma will be proved. The required inequality is obviously implied by the following more general situation, which is of use in other questions of this kind. Both are practically obvious, but we give a formal proof.

If $p>1$, and $h, x, h_{i}, x_{i}(i=1, \cdots, p)$ are any integers $>0$ such that (A) $x_{i} \geqq x(i=1, \cdots, p)$; (B) $x_{1}+\cdots+x_{p}>p x$; (C) $h x=h_{1} x_{1}+\cdots+h_{p} x_{p}$; then $h>h_{1}+\cdots+h_{p}$.

To prove this, define the $e_{i}$ by $x_{i} / x=1+e_{i}$. Then, from (A), $e_{i} \geqq 0$; from (B) $e_{1}+\cdots+e_{p}>0$; hence at least one of $e_{1}, \cdots, e_{p}$ is $>0$. From (C),

$$
1=\left(\frac{h_{1}}{h}+\cdots+\frac{h_{p}}{h}\right)+\left(e_{1} \frac{h_{1}}{h}+\cdots+e_{p} \frac{h_{p}}{h}\right) ;
$$

the second () is $>0$; hence

$$
1>\frac{h_{1}}{h}+\cdots+\frac{h_{p}}{h}, h>h_{1}+\cdots+h_{p} .
$$

The inequality is also easily seen from a simple contradiction. This completes the proof of the lemma.

3. Expansions of $\operatorname{sn} x, \operatorname{dn} x$. Proceeding as before from

$$
\begin{aligned}
& \operatorname{sn} x=\sum_{\bullet=0}^{\infty} \frac{(-1)^{s} P_{c}\left(k^{2}\right)}{(2 s+1) !} x^{2 s+1}, \quad P_{c}\left(k^{2}\right) \equiv \sum_{r=0}^{\infty} p_{r}(s) k^{2 r}, \\
& \vartheta_{2}^{2} \operatorname{sn}\left(x \vartheta_{3}^{2}\right)=\vartheta_{2} \vartheta_{3} \frac{\vartheta_{1}(x)}{\vartheta_{0}(x)}=4 \sum q^{m / 2}\left[\sum \sin t x\right] \\
& (m=1,3,5, \cdots ; m=t \tau, t>0, \tau>0),
\end{aligned}
$$

we get the recurrence (8) for the $p_{r}(s)$ :

$$
\sum_{r=0}^{\dot{2}} 2^{4 r} p_{r}(s) N(2 m, 4 s+4,4 r+2)=\zeta_{20+1}(m),
$$

where $\zeta_{20+1}(m)$ denotes the sum of the $(2 s+1)$ th powers of the divisors of $m$.

To illustrate the calculations, let $m=1,3,5,7$. Then

$$
p_{0}(s) N(2,4 s+4,2)=\zeta_{2 s+1}(1) ; \quad p_{0}(s)=1 \text {. }
$$


For $m=3,5,7$ we need the following $N$ 's:

$$
\begin{aligned}
N(2 m, 4 s+4,2 m) & =1 ; \\
N(6,4 s+4,2) & =\frac{2(4 s+2) !}{1 !(4 s+1) !}=8 s+4 ; \\
N(10,4 s+4,2) & =2+\frac{2^{2}(4 s+2) !}{2 !(4 s) !}=2\left(16 s^{2}+12 s+3\right) \\
N(10,4 s+4,6) & =\frac{2(4 s-2) !}{1 !(4 s-3) !}=4(2 s-1) ; \\
N(14,4 s+4,2) & =\frac{1 \cdot 2^{3}(4 s+2) !}{3 !(4 s-1) !}+2 \cdot 2 \cdot \frac{(4 s+2) !}{1 !(4 s+1) !} \\
& =\frac{8}{3}(2 s+1)\left(16 s^{2}+4 s+3\right) ; \\
N(14,4 s+4,6) & =\frac{2^{2} \cdot(4 s-2) !}{2 !(4 s-4) !}+6=32 s^{2}-40 s+18 \\
N(14,4 s+4,10) & =\frac{2(4 s-6) !}{1 !(4 s-7) !}=4(2 s-3) .
\end{aligned}
$$

It will be sufficient to indicate the origin of one of these, say $N(14,4 s+4,2)$ :

$$
14=2 \cdot 1^{2}+\left[3 \cdot 2^{2}+(4 s-1) 0^{2}\right]=1^{2}+3^{2}+\left[2^{2}+(4 s+1) 0^{2}\right] .
$$

Substituting these values in (8), and using $\zeta_{2++1}(3)=3^{2 s+1}+1$, etc., we find

$$
\begin{aligned}
p_{0}(s)= & 1 ; \quad 2^{4} p_{1}(s)=3^{2 s+1}-8 s-3 ; \\
2^{8} p_{2}(s)= & 5^{2 s+1}-4(2 s-1) 3^{2 s+1}+32 s^{2}-32 s-17 ; \\
2^{12} p_{2}(s)= & 7^{2 s+1}-4(2 s-3) 5^{2 s+1}+\left(32 s^{2}-88 s+30\right) 3^{2 s+1} \\
& -\frac{1}{8}\left(256 s^{8}-1056 s^{2}+752 s+471\right),
\end{aligned}
$$

agreeing with the values stated by Hermite. As in $\$ 2$, it can be shown that the general form is

$$
2^{4 i} p_{j}(s)=(2 j+1)^{2 s+1}+B_{1}(s)(2 j-1)^{2 s+1}+\cdots+B_{j}(s) 1^{2 s+1},
$$

where $B_{r}(s)$ is a polynomial in $s$ of degree $r$ with rational coefficients. From the MacLaurin expansion the $p_{j}(s)$ are integers. An explicit (determinant) form follows from (8). The relation $\mathrm{sn}(k x, 1 / k)=k \operatorname{sn}(x, k)$ gives $p_{i}(s)=p_{s-j}(s)$.

For $\operatorname{dn} x$ we have 


$$
\begin{aligned}
& \operatorname{dn} x=1+\sum_{\theta-1}^{\infty}(-1)^{\cdot} R_{\bullet}\left(k^{2}\right) \frac{x^{2 s}}{(2 s) !}, R_{\bullet}\left(k^{2}\right)=\sum_{j=0}^{i-1} r_{j}(s) k^{2 j+2} ; \\
& \vartheta_{8}^{2} \operatorname{dn}\left(x \vartheta_{8}^{2}\right)=\vartheta_{0} \vartheta_{3} \frac{\vartheta_{8}(x)}{\vartheta_{0}(x)}=1+4 \sum q^{n}\left(\sum(-1 \mid \tau) \cos 2 t x\right) \\
& (n=1,2,3, \cdots ; n=t \tau, \tau \text { odd } t>0, \tau>0) ; \\
& \sum_{j=0}^{n-1} 2^{4 j} N(4 n, 4 s+2,4 j+4) r_{j}(s)=2^{2 s-2} \xi_{2 s}(n),
\end{aligned}
$$

where $\xi$ is as defined in $\S 2$. To calculate the successive $r_{j}(s)$, or to exhibit a determinant for $r_{j}(s)$, we take $n=1,2,3, \cdots$, and proceed as before. Thus

$$
\begin{aligned}
& r_{0}(s)=2^{2 s-2} ; \quad r_{1}(s)=2^{2 s-6}\left(2^{2 s}-8 s+4\right) ; \\
& r_{2}(s)=2^{2 s-10}\left[3^{2 s}-4(2 s-3) 2^{2 s}+32 s^{2}-88 s+31\right] .
\end{aligned}
$$

The general form is

$$
r_{j}(s)=2^{2 s-4 j-2}\left[(j+1)^{2 s}+C_{1}(s) j^{2 s}+C_{2}(s)(j-1)^{2 s}+\cdots+C_{j}(s) 1^{\bullet}\right],
$$

where $C_{n}(s)$ is a polynomial of degree $n$ in $s$ with rational coefficients. The relation $\operatorname{dn}(k u, 1 / k)=\mathrm{cn}(u, k)$ gives $r_{j}(s)=q_{s-1-i}(s)(q$ as in $\$ 2)$; but this does not enable us to calculate the general $r_{j}(s)(j=1,2, \cdots)$ successively from the $q_{i}(s)$.

4. Reciprocal of $\operatorname{sn} x$. This will illustrate expansions in which the origin is a simple pole, and in which it is necessary to use the Bernoulli or Euler numbers to obtain the coefficients. From our paper already cited, ${ }^{*}$ we have

$$
\begin{aligned}
\frac{x \vartheta_{8}^{2}}{\operatorname{sn}\left(x \vartheta_{s}^{2}\right)}=x \vartheta_{2} \vartheta_{3} \frac{\vartheta_{0}(x)}{\vartheta_{1}(x)}=x \csc x+4 x \sum q^{n}\left[\sum \sin \tau x\right] \\
\quad(n=1,2,3, \cdots ; n=t \tau, t>0, \tau>0, \tau \text { odd }),
\end{aligned}
$$

the multiplier $x$ being introduced to render the series regular at the origin. The form of the MacLaurin series is easily seen from the indicated division of $x$ by the power series for sn $x$ in $\S 3$, and we have

$$
\frac{x}{\operatorname{sn} x}=1+\sum_{s=1}^{\infty} \frac{x^{2 s}}{(2 s-1) !} H_{s}\left(k^{2}\right) ; \quad H_{o}\left(k^{2}\right)=\sum_{r=0}^{\infty}(-1)^{r} h_{r}(s) k^{2 r} .
$$

To expand $x \csc x$ we shall use the numbers $R$ of Lucas $\dagger$ defined by the symbolic identity

- Messenger of Mathematics, vol. 54 (1924), pp. 116-176, \$14, p. 172.

$\dagger$ E. Lucas, Theorie des Nombres, chapter xiv. 


$$
x \csc x=2 \cos R x=2 \sum_{0=0}^{\infty} \frac{(-1)^{s} x^{2 s}}{(2 s) !} R_{2 s} .
$$

In terms of the Bernoulli numbers $B$, in the even-suffix notation, $x \operatorname{ctn} x=\cos 2 B x, \quad B_{0}=1, \quad B_{1}=-\frac{1}{2}, \quad B_{2}=\frac{1}{6}, \quad B_{4}=-\frac{1}{30}, \ldots$, we have

$$
R_{2 \mathrm{~s}}=\left(1-2^{2 \mathrm{~s}-1}\right) B_{2 \mathrm{~s}}, \quad R_{0}=\frac{1}{2}, \quad R_{2}=-\frac{1}{6}, \quad R_{\mathrm{t}}=\frac{7}{30}, \ldots .
$$

Proceeding as before we find

$$
s v_{3}^{4 s} H_{\bullet}\left(k^{2}\right)=(-1)^{\circ}\left[R_{2 s}-4 s \sum_{n=1}^{\infty} q^{n} \zeta_{20-1}^{\prime}(n)\right],
$$

where $\zeta_{20-1}^{\prime}(n)$ denotes the sum of the $(2 s-1)$ th powers of all the odd (positive) divisors of $n$. The left member is

$$
s\left[h_{0}(s) \vartheta_{2}^{4}+\sum_{r=1}^{\dot{s}} h_{r}(s) \vartheta_{2}^{4 r} \vartheta_{2}^{40-4 r}\right] ;
$$

and

$$
\vartheta_{3}^{40}=1+\sum_{n=1}^{\infty} q^{n} N(n, 4 s),
$$

where $N(n, 4 s)$ denotes the total number of representations of $n$ as a sum of $4 s$ squares. Hence

$$
\begin{gathered}
\operatorname{sh}_{0}(s)=(-1)^{s} R_{20}, \\
h_{0}(s) N(n, 4 s)+\sum_{r=1}^{s}(-1)^{r} h_{r}(s) 2^{4 r} N(4 n, 4 s, 4 r)=-4(-1) \cdot \zeta_{28-1}^{\prime}(n),
\end{gathered}
$$

from which the successive $h_{j}(s)$ can be calculated as in previous examples, and the general form is easily determinable.

5. Expansion of $\varphi(x)$. This is referred to the expansion of $x^{2} / \operatorname{sn}^{2} x$ by means of

$$
x^{2} \varphi\left(x ; g_{2}, g_{3}\right)=\frac{x^{2}}{\operatorname{sn}^{2}(x, k)}-\frac{1+k^{2}}{3} x^{2},
$$

in the customary notation. As Gruder (loc. cit., \$13) has shown the connection between the coefficients in the polynomials (in $g_{2}, g_{3}$, or in the absolute invariant $\left.g_{2}{ }^{3} / g_{3}{ }^{2}\right)$ occurring as coefficients in the power series for $\varphi\left(x ; g_{2}, g_{3}\right)$ 
and the coefficients in the polynomials (in $k^{2}$ ) occurring as coefficients in the expansion of $x^{2} / \mathrm{sn}^{2}(x, k)$, it will suffice here to give the recurrence for the latter.

From the expansion of $x / \operatorname{sn} x$ it is easily seen that the MacLaurin series is of the form

$$
\frac{x^{2}}{\operatorname{sn}^{2} x}=\sum_{s=0}^{\infty} \frac{x^{2 s}}{(2 s) !} T_{2 s}\left(k^{2}\right), T_{2 s}\left(k^{2}\right)=\sum_{r=0}^{\infty} t_{r}(s) k^{2 r}, T_{0}\left(k^{2}\right)=1 ;
$$

and from the author's paper cited above, ${ }^{*}$ we have

$$
\begin{aligned}
\frac{x^{2} \vartheta_{3}^{4}}{\operatorname{sn}^{2}\left(x \vartheta_{z}^{2}\right)}= & x^{2} \vartheta_{2}^{2} \vartheta_{3}^{2} \frac{\vartheta_{0}^{2}(x)}{\vartheta_{1}^{2}(x)}, \\
= & x^{2}\left[4 \sum q^{n} \sigma_{1}(n)+\csc ^{2} x-8 \sum q^{2 n}\left(\sum d \cos 2 d x\right)\right] \\
& (n=1,2,3, \cdots ; n=d \delta, d>0, \delta>0),
\end{aligned}
$$

where $\sigma_{1}(n) \equiv \zeta_{1}(n)+\zeta_{1}^{\prime}(n), \zeta, \zeta^{\prime}$ being as defined in $\$ \$ 3,4$. To expand $x^{2} \csc x^{2}$ we may use the Bernoulli numbers of the second order, $\dagger$ or proceed as follows to obtain the coefficients at once in terms of ordinary Bernoulli numbers. The symbolic identity defining the Bernoulli numbers $B$ is $x \operatorname{ctn} x=\cos 2 B x$. Differentiating this with respect to $x$ and multiplying the result throughout by $x$, we get

$$
x^{2} \csc ^{2} x=\cos 2 B x+2 B x \sin 2 B x .
$$

Hence, equating coefficients of like powers of $x$, we have

$$
x^{2} \csc ^{2} x \equiv \cos D x, D_{3 n}=2^{2 n}(1-2 n) B_{2 n} \quad(n=0,1, \cdots) .
$$

The rest of the work is like that in preceding sections, and we get (from the coefficients of $x^{2}, x^{2}, s>1$, in the identity between power series in $x$ ) the preliminary results

$$
\begin{aligned}
\vartheta_{2}^{4} T_{2}\left(k^{2}\right) & =8\left[\sum q^{n} \sigma_{1}(n)-2 \sum q^{2 n} \zeta_{1}(n)\right]-D_{2}, \\
\vartheta_{2}^{4} T_{2 s}\left(k^{2}\right) & =(-1)^{\circ}\left[D_{2 s}+16 s(2 s-1) \sum q^{2 n} 2^{2 s-2} \zeta_{2 s-1}(n)\right.
\end{aligned}
$$

for $s>1$, the summations referring to $n=1,2,3, \ldots$. The first of these gives

$$
t_{0}(1) \vartheta_{2}^{4}+t_{1}(1) \vartheta_{2}^{4}=8\left[\sum q^{n} \sigma_{1}(n)-2 \sum q^{2 n} \zeta_{1}(n)\right]-D_{2} ;
$$

and there are the known expansions

$$
\begin{array}{r}
\vartheta_{3^{4}}^{4}=1+8 \sum q^{n}(-1)^{n} \lambda_{1}(n), \vartheta_{2}^{4}=16 \sum q^{m} \zeta_{1}(m) \\
(n=1,2,3, \cdots ; m=1,3,5, \cdots),
\end{array}
$$

* Messenger of Mathematics, vol. 54 (1924), pp. 116-176; $\$ 16$, p. 173.

$\dagger$ N. E. Nörlund, Differenzenrechnung, 1924, p. 129, et seq. The symbolic processes used here are justified (among other places) in my Algebraic Arithmetic, 1927. 
where

$$
\lambda_{1}(n)=\left[1+2(-1)^{n}\right] \zeta_{1}^{\prime}(n) .
$$

From the definitions of the functions it is easily seen that

$$
\sum q^{n} \sigma_{1}(n)-2 \sum q^{2 n} \zeta_{1}(n)=2 \sum q^{n} \zeta_{1}^{\prime}(n) .
$$

Hence, finally, we get

$$
t_{0}(1)=t_{1}(1)=\frac{2}{3} \text {. }
$$

All this detail for $t_{0}(1), t_{1}(1)$ is of course unnecessary, as $T_{2}\left(k^{2}\right)$ is readily seen to be $\frac{2}{3}\left(1+k^{2}\right)$; but the reduction provides a check on the expansions.

Reducing the second of the above preliminary results as before we find

$$
\begin{gathered}
t_{0}(s)=(-1)^{\bullet} D_{2 s}=(-1)^{\cdot} 2^{2 s}(1-2 s) B_{2 s} \\
t_{0}(s) N(m, 4 s)+\sum_{r=1}^{m} 2^{4 r} t_{r}(s) N(4 m, 4 s, 4 r)=0
\end{gathered}
$$

$$
(m=1,3,5, \cdots) \text {; }
$$

$$
\begin{aligned}
t_{0}(s) N(2 n, 4 s)+\sum_{r=1}^{2 n} 2^{4 r} t_{r}(s) N(8 n, 4 s, 4 r) . \\
\quad=(-1)^{\circ} s(2 s-1) 2^{2 s+2} \zeta_{2 s-1}(n) \quad(n=1,2,3, \cdots),
\end{aligned}
$$

all of which hold only for $s>1$. The functions $N, \zeta$ are as previously defined. From these the structure of $t_{j}(s)$ is seen as before (the few specimens given by Hermite, Oeuvres, vol. 3 , p. 239, in another notation, do not indicate that the $(2 s-1)$ th powers of integers $>1$ enter the $t_{r}(s)$ for $\left.r>2\right)$. Taking $m=1$ in (15) we get $(s>1)$

$$
t_{1}(s)=(-1)^{s} s(2 s-1) 2^{2 s-1} B_{2 s,}
$$

and $n=1$ in (16),

$$
t_{2}(s)=(-1)^{s} s(2 s-1) 2^{2 s-6}\left[1-2(4 s-7) B_{2 s}\right],
$$

which check with tabulated results for $s=2,3,4,5$.

6. Further developments. Hermite (Oeuvres, vol. 3, p. 245) was interested in these expansions partly on account of their possible applications to Gyldèn's methods (followed by Brendel) in the computation of perturbations, particularly for the so-called critical planets, whose mean motion is almost commensurable with Jupiter's. In this connection the expansions of powerproducts of $\operatorname{sn} x, \operatorname{cn} x, \operatorname{dn} x$ are required, the powers being positive or negative. From the series for $\operatorname{sn} x, \operatorname{cn} x, \operatorname{dn} x$ and their reciprocals, the general $k^{2}$-polynomial form of the coefficient of $x^{n}$ in the expansion of $\operatorname{sn}^{a} x \operatorname{cn}^{b} x \mathrm{dn}^{c} x$, 
where $a, b, c$ are integers, can be inferred. The general trigonometric series for use in the present method were investigated by Meyer, ${ }^{*}$ from whose general results the types of arithmetical functions appearing in the coefficients can be determined. As this is quite an extensive subject we shall not go into it here, except to note a necessary change which occurs in the arithmetical character of the coefficients when any one of $a, b, c$ passes the value $2:$ the functions are no longer expressible in terms of the divisors of $a$ single integer (as they are for all the expansions in the present paper), but refer to representations in quadratic forms other than $x y$ (which introduces the functions of divisors). For example, one function is $\sum(x y z w)^{4}$, the sum being taken over all representations of a fixed integer in the form $x^{2}+y^{2}+z^{2}+w^{2}$. This is analogous to the similar situation concerning the number of representations of an integer as a sum of $2 s$ squares when $s>4$, where we have the classical theorems for $s=5,6$ which introduce quadratic forms other than $x y . \dagger$

* C. O. Meyer, Crelle's Journal, vol. 37 (1848), pp. 273-304.

$\dagger$ For the following references to the astronomical applications, I am indebted to Professors A. O. Leuschner and R. H. Sciobereti.

(1) M. Brendel, Abhandlungen der Königlichen Gesellschaft der Wissenschaften zu Göttingen, vol. 1, No. 2 (1898), part 1, pp. 45-51 and pp. 53-55; vol. 6, No. 4 (1909), part 2, chapter 2, p. 12.

(2) H. Gyldèn, Studien auf dem Gebiete der Störungstheorie, Academy of St. Petersburg, Memoirs, (7), vol. 16.

(3) F. Tisserand, Mécanique Céleste, vol. I, Chapter XVII: Sur certaines fonctions des grands axes qui se presentent dans le développement de la fonction perturbatrice.

(4) H. Poincare, Les Méthodes Nouvelles de la Mécanique Céleste. Poincarés summary of Gyldèn's method is in vol. 2, p. 202, et seq., more particularly pp. 247-251-253.

(5) H. Gyldèn, Traité des Orbites Absolues des 8 Planètes Principales, 1893. vol. I, book II, chapter II; vol. I, book III, chapter II, p. 357, p. 394. Brendel's work on this particular subject is merely a reproduction of Gyldèn's treatment of the perturbative function.

California Institute of Technology, Pasadena, Calit. 